\title{
Future Suicide Attempt and Responses to Serotonergic Challenge
}

\author{
John G Keilp*, 1,2, Maria A Oquendo, ,2, Barbara H Stanley,2, Ainsley K Burke ${ }^{1,2}$, Thomas B Cooper ${ }^{1,2}$, \\ Kevin M Malone ${ }^{1,2}$ and J John Mann ${ }^{1,2}$ \\ 'Department of Neuroscience, New York State Psychiatric Institute, New York, NY, USA; ${ }^{2}$ Department of Psychiatry, Columbia University College \\ of Physicians and Surgeons, New York, NY, USA
}

\begin{abstract}
Blunted neurohormonal responses to serotonergic agents are found in major depression and suicidal behavior, but there have been no prospective studies of their relationship to later suicide attempt. In this study, healthy volunteers and depressed subjects were administered a fenfluramine (FEN) and placebo challenge test at baseline and then followed for 2 years. Seven subjects made suicide attempts within the follow-up period. Healthy volunteers, depressed non-attempters, depressed past suicide attempters, and depressed future attempters were compared on plasma prolactin and cortisol responses, as well as on mood (Profile of Mood States; POMS) and behavioral measures that were assessed at baseline and at the end of each challenge testing day. Both past and future attempters had lower total prolactin output (area under the curve) in response to FEN relative to non-patients. Future attempters had lower cortisol response relative to all other groups. All subject groups reported a decrease in POMS Fatigue subscale score and increase in finger tapping rate after receiving FEN. Depressed subjects reported a significant decline in POMS Total, Depression, and Tension/Anxiety scores, but future attempters' did not, showing a slight mean increase. Lower cortisol response correlated with greater suicidal ideation 3 months and I year post-study. Logistic regression revealed that blunting of cortisol response and worsening of mood after FEN, and younger age could be used to predict later suicide attempt in the majority of cases (4/7). Results suggest that blunted cortisol and unfavorable acute mood response to serotonergic challenge, in the context of the general activating effects of these drugs, may be a risk factor for later suicide attempt.
\end{abstract}

Neuropsychopharmacology (2010) 35, 1063-1072; doi:I0.1038/npp.2008.28; published online 19 March 2008

Keywords: serotonin; cortisol; prolactin; challenge test; suicide; depression

\section{INTRODUCTION}

Pharmacologic challenge paradigms have been used extensively to investigate the responsiveness and integrity of the central serotonin system. They have primarily been used to study depression and personality disorder, including traits associated with impulsive/aggressive behaviors and suicidal behavior. Blunted prolactin response to these challenge agents - such as fenfluramine (FEN) or citalopram - has been found in most studies of major depression (Newman et al, 1998), as well as in personality disorders characterized by impulsive and aggressive traits (New et al, 2004). Blunting of the prolactin response correlates with impulsiveness and aggressiveness in both patient (Coccaro et al, 1989; Soloff et al, 2003) and non-patient populations

\footnotetext{
*Correspondence: Dr JG Keilp, New York State Psychiatric Institute, Box 42, I05I Riverside Drive, New York, NY 10032, USA,

Tel: + | 2125435866, Fax: + | 2125436017,

E-mail: jgk।3@columbia.edu

Received 18 September 2007; revised 23 January 2008; accepted 24 January 2008
}

(Manuck et al, 1998, 2002). Given the association with both depression and aggression, it is not surprising that a blunted prolactin response to FEN is also associated with past suicidal behavior (Cleare et al, 1996; Malone et al, 1996; Correa et al, 2000; Duval et al, 2001; Soloff et al, 2003), consistent with a variety of other measures of central serotonin function (Mann, 2003).

Although the majority of these studies have focused on the prolactin response to serotonergic challenge, other hormones released by serotonergic stimulation of the hypothalamus/pituitary have been measured as well, including adrenocorticotrophic hormone (ACTH) and growth hormone, as well as ACTH-stimulated cortisol secretion. In studies using FEN, other hormone responses typically do not discriminate clinical groups as well as prolactin (Newman et al, 1998). In the case of cortisol, this may be due in part to methodological factors, since these challenge tests tend to be run early in the day when circulating levels of cortisol are at their highest and declining. As a result, administration of challenge agents results in an attenuation of this circadian decline rather than a clear increase from baseline levels (Newman et al, 
1998). Nonetheless, blunting of cortisol response has been found in suicide attempters (Cleare et al, 1996; Duval et al, 2001). Cortisol response to serotonergic challenge may have particular relevance to the assessment of acute psychopathology, since prolactin responses to these challenge agents tend to be stable before and after treatment (Flory et al, 1998; Dulchin et al, 2001), while cortisol response is not (Bhagwagar et al, 2002b).

These differences in the nature of prolactin and cortisol responses may be important for the assessment of later risk for suicidal behavior. Prospective studies suggest that risk for both future attempt and completion may be evident in baseline measures of serotonin activity or hypothalamicpituitary-adrenal (HPA) axis dysregulation (Mann and Currier, 2007). FEN and other serotonin challenge paradigms provide indices of both serotonin system responsiveness and HPA axis function and may be able to predict risk of suicide attempts in major depression.

Beyond these biological effects, mood and behavioral effects of serotonergic challenge agents may also have prognostic significance. In a small sample of subjects with familial depression who received fluorodeoxyglucose positron emission tomography (PET) scans after the administration of FEN, we found an acute reduction in self-reported depressed mood that correlated with reduced glucose metabolism in the ventromedial prefrontal cortex (Kegeles et al, 2003), within a network of regions typically associated with treatment responsiveness. Positive initial mood responses to serotonergic agents, as early as the first week of treatment, appear related to treatment efficacy (Taylor et al, 2006). Conversely, a poor initial mood response to treatment, as well as increasing activation, may be associated with the persistence or even emergence of suicidal ideation (Perlis et al, 2007).

In this study, we analyzed data from FEN challenge tests that had been administered to drug-free healthy volunteers, drug-free depressed subjects with no history of suicide attempt, and depressed subjects who had made one or more suicide attempts in the past. These challenge procedures included measures of both mood and motor speed. We subsequently followed subjects for 2 years, and identified a subsample that made a suicide attempt after the initial assessment. We compared these future attempters' neuroendocrine, mood, and behavioral responses during the FEN challenge test with healthy volunteers, depressed past suicide attempters, and depressed patients who had never made a suicide attempt. We hypothesized that future suicide attempters would show exaggerated blunting of both prolactin and cortisol responses after FEN administration. We conducted exploratory studies of effects of serotonin release on mood and motor speed, and relationship of those responses to outcome on follow-up.

\section{METHODS}

\section{Subjects}

Groups were (1) 15 healthy volunteers (non-patient comparison group); (2) 19 depressed subjects without any history of suicidal behavior (non-attempters); (3) 19 depressed subjects with a past history of suicidal attempt (past attempters) and no future attempt; and (4) 7 depressed subjects who would go on to make a suicide attempt within the 2 years following the assessment (future attempters). Six of the seven subjects who went on to make an attempt had made an attempt in the past. One of these future attempts was a completed suicide.

\section{Clinical Assessments}

Psychiatric illnesses were ruled out in non-patients using the non-patient version of the Structured Clinical Interview for DSM-IV (SCID-NP; First et al, 1997). Diagnosis was established in patients using the SCID, Axes I (SCID-I; Spitzer et al, 1990) and II (SCID-II; First et al, 1996). History of past suicidal behavior was assessed via semistructured interview using the Columbia Suicidal Behavior History Form (Oquendo et al, 2003c), which was repeated at 3 months, 1 year, and 2 years after discharge.

Baseline clinical assessments included the Hamilton Depression Scale (Hamilton, 1960), Beck Depression Inventory (Beck et al, 1961), Global Assessment of Functioning, Beck Hopelessness Scale (Beck et al, 1974), Scale for Suicidal Ideation (Beck et al, 1979), Barratt Impulsiveness Scale (Barratt, 1985, 1994), Buss-Durkee Hostility Inventory (Buss and Durkee, 1961), and BrownGoodwin Aggression History (Brown et al, 1979). For subjects with a past history of suicide attempt, the Beck Suicide Intent Scale (Beck et al, 1975) and a rating of Medical Lethality of past attempts (op. cit.) was also completed.

A set of repeatable assessments was also completed at baseline and during the course of the FEN challenge procedure. Repeated assessments included the Profile of Mood States (POMS; McNair et al, 1981), a 65-item questionnaire that produces a Total mood disturbance score and six subscale scores for Depression, Tension/ Anxiety, Anger/Hostility, Confusion, Fatigue, and Vigor. Subjects also received a brief assessment of motor speed via the finger tapping test, a standard neuropsychological measure using a telegraph-like key and counter to assess speed of intentional finger oscillations (Reitan and Wolfson, 1985). Drug side effect ratings for activation, dizziness, nausea, and confusion (0-3 rating: none, mild, moderate, and severe) were completed by examiners on each challenge day.

\section{FEN Challenge}

The FEN challenge involved a placebo and active drug day, done in a fixed order, as described previously (Kegeles et al, 2003; Oquendo et al, 2003d). Placebo or $0.8 \mathrm{mg} / \mathrm{kg}$ D, L FEN ( $\sim 60 \mathrm{mg}$ for average subject) was administered at 0800 hours, blood samples were drawn $15 \mathrm{~min}$ before drug administration, immediately after drug administration (hour 0 ) and at hourly intervals for the next $5 \mathrm{~h}$. Bloods were assayed for prolactin, cortisol, FEN and norfenfluramine levels. Prolactin and cortisol levels were determined by immunoradiometric assay, drug and metabolite levels by gas liquid chromatography, according to methods published previously (Kegeles et al, 2003; Oquendo et al, 2003d).

Mood and behavioral assessments were completed on the day before the initial FEN procedure (run-in baseline to 
reduce practice effects on challenge days), and at the end of the procedure on each of the two challenge days $(5.5 \mathrm{~h}$ after FEN administration). Medication side effect ratings were completed at the start of these assessments on each of the two challenge days only. Because all subjects were participants in a study examining the effects of FEN challenge on cerebral glucose metabolism and received a PET scan between hours 3 and 5 of the standard FEN procedure (Kegeles et al, 2003; Oquendo et al, 2003d), they were unavailable for behavioral testing at this time.

\section{Statistical Analyses}

Demographic and baseline clinical variables were compared using one-way ANOVA and post hoc Tukey LSD comparisons for continuous variables, $\chi^{2}$ for categorical variables. Baseline, peak change from baseline, and total area under the curve (AUC; Pruessner et al, 2003) in prolactin and cortisol (FEN day only) were compared among groups using analysis of covariance (ANCOVA) within general linear models, with age, sex, and combined FEN/norfenfluramine level at peak (hours 3 and 4, averaged) as covariates. These three parameters effectively characterize the overall response curves for each group. Peak response was of primary interest, but AUC was analyzed to capture responses that might be modest but prolonged over the course of several hours. Peak change was computed as the difference between the nadir up to hour 1 of the procedure, and the highest point thereafter. Mood ratings derived from the POMS were analyzed with repeated-measures ANCOVA across study days, with age and sex as covariates. Finger tapping scores were converted to normatively corrected $z$-scores - which adjust for age and sex effects - based on published external normative data (Spreen and Strauss, 1998), and averaged across both hands. Finger tapping scores and side effect ratings were compared using repeated-measures ANOVA under general linear models.

The fixed order of presentation of placebo and drug days limits our ability to attribute changes in mood or performance to drug effects $v s$ accumulated order effects. However, repeated assessment effects were managed, in part, by the use of a run-in baseline. We attributed changes in mood ratings or behavioral performance to drug effects if the overall time or group by time effect in repeatedmeasures analysis was significant, and if there was a significant difference in ratings or performance between drug and placebo days (significant differences between drug day and baseline alone were not attributed to drug effects).

Correlations were computed among scores that differentiated groups, and logistic regression was used to determine if neurohormonal response and mood change could be used to predict later suicide attempt.

\section{RESULTS}

\section{Demographic and Clinical Characteristics of Groups}

Both past and future attempters were younger than nonpatients, but all groups were comparable in education, WAIS-R Vocabulary score, and gender distribution (Table 1). The depressed groups were comparably depressed on the Hamilton Depression Rating Scale, although past and future attempters had higher Beck Depression Scale scores as we have previously reported in larger samples (Mann et al, 1999; Grunebaum et al, 2005). There was no greater frequency of bipolar depression, borderline personality, substance use disorder, or posttraumatic stress disorder in the future attempters group relative to other patient groups. The only distinguishing clinical characteristic of the future attempters was higher current suicidal ideation and Beck Depression scores.

Future suicide attempts were made, on average, $18.7 \pm 7.4$ months after FEN assessments, with the earliest at 5 months. One subject made multiple attempts (three) and made the earliest attempt. Maximum lethality rating of future attempts averaged $3.4 \pm 2.8$, and ranged from 0 (no injury) to 8 (death). Suicidal ideation remained high in future attempters $(10.6 \pm 12.0$ at 3 months post-discharge, $14.7 \pm 9.2$ after 1 year) and consistently higher than other depressed subjects $(\mathrm{F}(2,33)=4.59, p=0.02)$. Beck Depression score also remained high $(26.0 \pm 17.6$ and $31.8 \pm 8.6)$ and higher than other groups $(\mathrm{F}(2,37)=6.24, p=0.005)$. Hamilton score remained high, but was comparable to other groups $(20.6 \pm 14.1$ and $27.6 \pm 8.9 ; \mathrm{F}(2,39)=2.29, p=0.11)$.

\section{FEN Challenge}

Raw prolactin and cortisol values in each group on the FEN day are presented in Figure $1 \mathrm{a}$ and $\mathrm{b}$.

Adjusted baseline $(\mathrm{F}(3,53)=0.93, p=0.43)$ and change to peak prolactin levels $(\mathrm{F}(3,51)=2.24, p=0.10)$ were not different among the groups (Table 2). AUC for prolactin, however, was significantly lower in both past and future attempters relative to non-patients $(\mathrm{F}(3,51)=3.88, p=0.01)$. Sex was, as expected, a significant covariate for all prolactin measures (for baseline: $\mathrm{F}(1,51)=9.97, p=0.003$; for change to peak: $F(1,51)=36.45, \quad p<0.001$; for AUC: $\mathrm{F}(1,51)=43.86, p<0.001)$, age affected both baseline prolactin $(\mathrm{F}(1,51)=7.26, p=0.009)$ and $\mathrm{AUC}(\mathrm{F}(1,51)=13.28$, $p=0.001$ ), both of which were lower in older subjects.

Adjusted baseline cortisol levels $(\mathrm{F}(3,54)=1.45, p=0.24)$ and $\operatorname{AUC}(\mathrm{F}(3,52)=0.71, p=0.55)$ were comparable among the groups; however, change to peak in cortisol $(\mathrm{F}(3,52)=3.88, p=0.01)$ was significantly lower in future attempters relative to all other groups (Table 2). These measures were not affected by sex of subject, and subject's age only affected AUC, which was higher in older subjects $(\mathrm{F}(1,52)=6.29, p=0.02)$.

As an additional check on these findings, baseline, change to peak, and AUC were computed for prolactin and cortisol on the placebo day. Each value was then used as a covariate in the analysis of the FEN day response (in addition to age, sex, and drug levels), as a control for non-pharmacologic effects on these outcome measures. Group differences were similar to those in the previous analyses. AUC for prolactin differed among groups $(\mathrm{F}(3,50)=4.57, p=0.007)$ with past and future attempters lower than non-patients (post hoc $p$ 's $<0.01$ ), and peak change in cortisol differed among groups $(\mathrm{F}(3,51)=4.58, p=0.007)$ with future attempters lower than all other groups (post hoc p's $<0.004$ ).

Supplemental analyses to examine the effect of unipolar $v s$ bipolar depression revealed no differences between these depression subtypes. Additional covariance analyses examining the effect of depression severity, using either the 
Table I Demographic and Clinical Rating Data



${ }^{\mathrm{a} O m n i b u s}$ ANOVA for continuous variables and $\chi^{2}$ for categorical variables.

${ }^{b}$ Tukey LSD post hoc test for continuous variables.

Hamilton scale score or the Beck Depression Inventory score, did not alter the nature of group differences.

\section{Mood and Behavior Measures}

On the POMS (Table 3 ), there were significant group effects for the Total score $(F(3,53)=10.86, p<0.001)$ and all subscales throughout the study (for all scales, $\mathrm{F}(3,53)>4.40, \quad p<0.01)$. All patients had significantly poorer scores than non-patients on all POMS measures throughout the study (all post hoc $p<0.02$ ). Future attempters were significantly worse than all other groups on the Depression subscale $(\mathrm{F}(3,53)=1.02, \quad p<0.001$, post hoc p's $<0.05$ ).

Significant change across evaluation day independent of group membership was found for the Fatigue subscale $(\mathrm{F}[2,106]=4.05, p=0.02)$, which worsened significantly on the placebo day, and then improved significantly on the FEN day.
Differential changes across evaluation days, across groups were found on the POMS Total $(\mathrm{F}(6,106)=2.42, p=0.03)$, Depression $(\mathrm{F}(6,106)=2.76, p=0.02)$, and Tension/Anxiety $(\mathrm{F}(6,106)=2.40, p=0.03)$ scales, with a near significant effect on the Anger/Hostility scale $(F(6,106)=2.01$, $p=0.07)$. In all cases, there was improvement in mood ratings in depressed non-attempters and past suicide attempters and a slight worsening of mood in the future attempters. Change scores computed as FEN day mood ratings minus placebo day ratings differed significantly across the groups (for POMS Total: $\mathrm{F}(3,53)=4.10, p=0.01$; for Depression: $\mathrm{F}(3,53)=3.68, p=0.02$; for Tension/Anxiety: $\mathrm{F}(3,53)=4.81, p=0.005$; all post hoc $p<0.02$ for comparisons of change scores between future attempters and either past attempters or depressed non-attempters). The nature of these differences on the POMS Total score (adjusted for sex and age) is illustrated in Figure 2.

On the finger tapping test (Table 3), performance did not differ among the groups at baseline and throughout the study, but changed significantly in all groups over the 

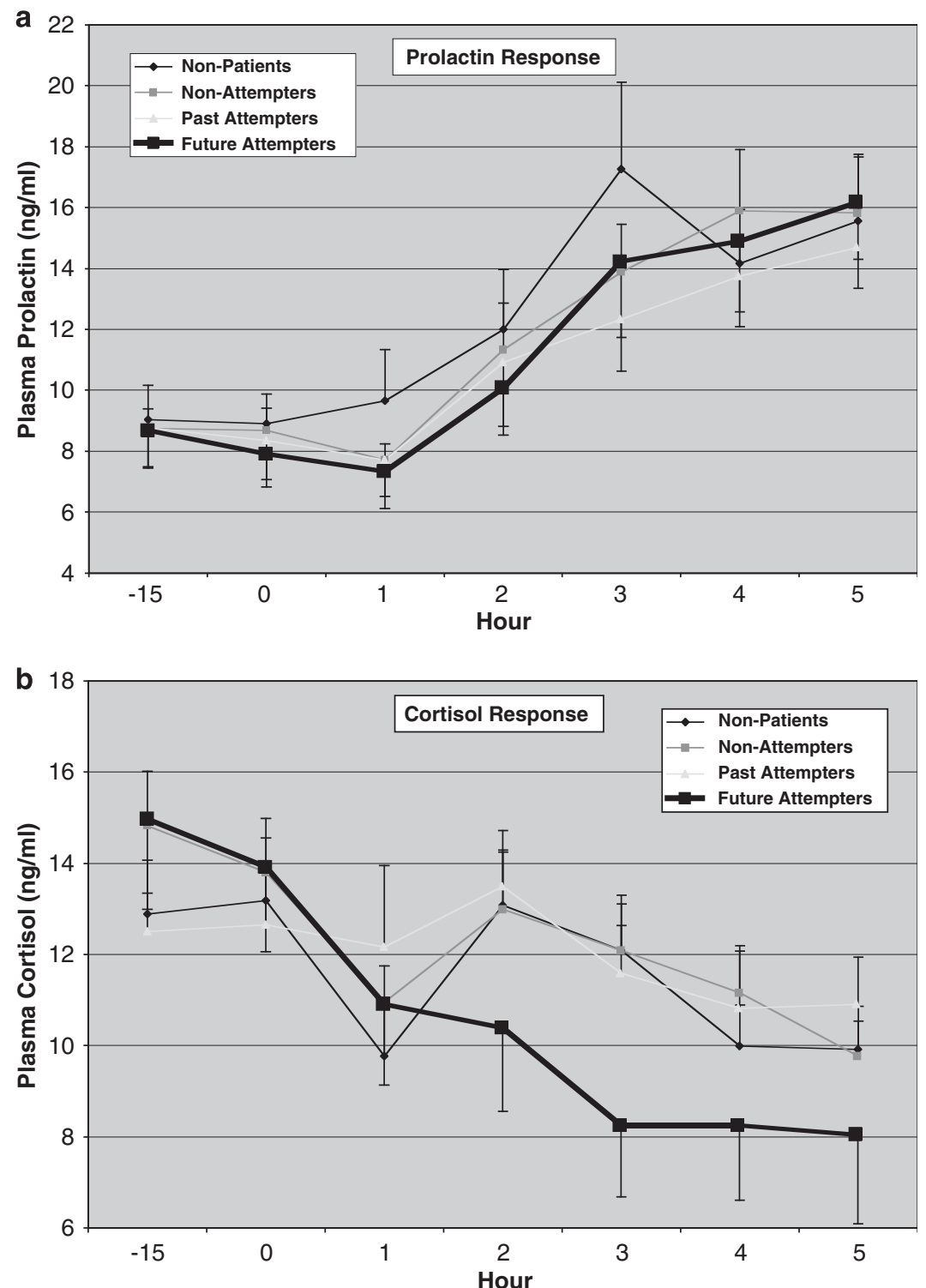

Figure I (a, b) Raw plasma prolactin and cortisol values (mean \pm SEM) for each group at initial baseline $(-15$ min) and at hourly intervals after administration of fenfluramine (active drug day).

course of the FEN challenge procedure $(\mathrm{F}(2,96)=12.05$, $p<0.001)$. Tapping rate was significantly higher on the FEN day than on either placebo or baseline days.

Overall, subjects showed a decrease in POMS Fatigue and increase in finger tapping rate on the FEN day relative to placebo. Patient subjects, with the exception of future attempters, showed an improvement in POMS Depression, Anxiety, and Total scores on the FEN day. Depression score, in fact, worsened in most future attempters $(5 / 7,71.4 \%)$, a significantly higher percentage than in the non-patient $(3 / 15,20.0 \%)$ non-attempter $(5 / 19,26.3 \%)$ or past attempter $(2 / 18,11.1 \%)$ groups $\left(\chi^{2}(3)=10.0, p=0.02\right)$.

\section{Side Effect Ratings}

Side effect ratings are presented in Table 3. Side effects of activation and nausea increased on the FEN day, but with no differences among subject groups. Change in activation ratings was due to mild increase in $17.2 \%(n=10)$ of all subjects (6 patients/4 non-patients); change in nausea was due to mild symptoms in $19.0 \%(n=11)$ of all subjects (7 patients/4 non-patients). Apparent activation was related to improvement in POMS Depression score in patients $(t(41)=2.28, p=0.03)$, but not finger tapping change $(t(39)=0.23, p=0.82)$, suggesting it was a proxy for a brightening of mood.

\section{Correlations}

AUC for prolactin (corrected for age, sex, and drug levels) was not associated with other variables, including the change to peak cortisol $(r=-0.01, p=0.95)$.

The peak change in cortisol response in patients was marginally associated with baseline suicidal ideation $(r=-0.28, p=0.06)$. The peak change in cortisol was also associated with suicidal ideation in the entire depressed 
Table 2 Hormonal and Drug Level Measures during FEN Challenge

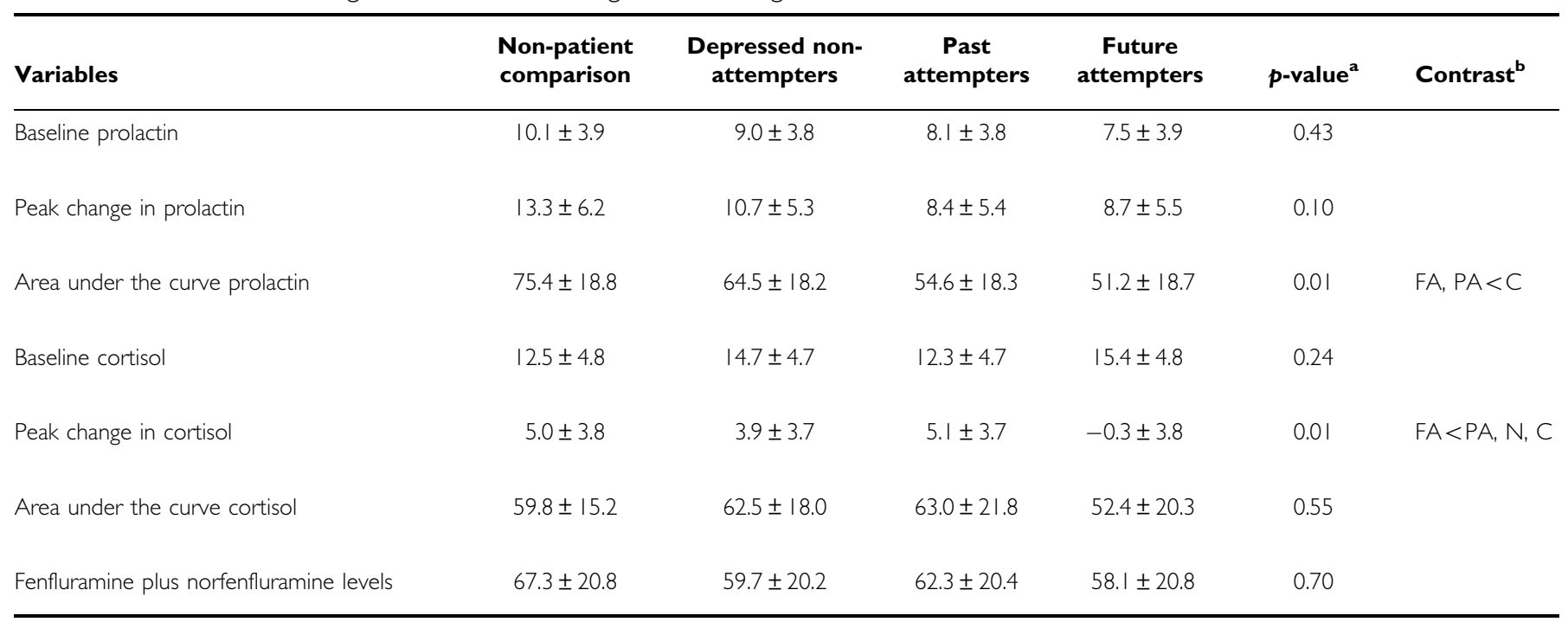

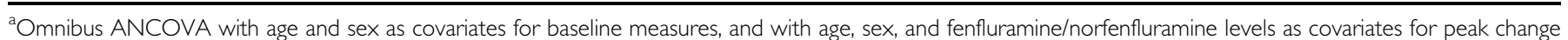
and area under the curve.

bTukey LSD post hoc test.

sample at both 3 months $(r=-0.32, p=0.05)$ and at 1 year $(r=-0.40, p=0.007)$. These associations approached significance in the future attempter group $(r$ 's $=-0.68, p=0.09$ at both 3 months and 1 year). It correlated as well with lifetime number of suicide attempts (log-transformed) among all attempters $(r=-0.39, p=0.05)$.

A supplemental, stepwise logistic regression was run in all depressed subjects to determine if a combination of variables derived from the challenge procedure might be useful in identifying future attempters. Change to peak cortisol, change in POMS Total score from placebo to FEN day, age, sex, and current level of suicidal ideation were used in the pool of predictors, with a $p$-to-enter of 0.05 . The final equation included three variables: lower change to peak cortisol (coefficient $=0.61$, Wald statistic $=4.28$, $p=0.04$, odds ratio $=1.84,95 \% \mathrm{CI}=1.03-3.28)$, less improvement in POMS Total score (coefficient $=0.10$, Wald statistic $=4.26, p=0.04$, odds ratio $=1.11,95 \% \mathrm{CI}=1.01-$ 1.22 ), and younger age (coefficient $=0.21$, Wald statistic $=3.69, p=0.05$, odds ratio $=1.24,95 \% \mathrm{CI}=1.00$ 1.54). This equation identified $57.1 \%$ of the future attempters (4/7), with a $2.3 \%(1 / 37)$ misclassification rate of other depressed subjects.

\section{DISCUSSION}

Lower AUC for prolactin response distinguished both past and future attempter groups from non-patients. This difference was not reflected in the peak change measure due to persistence of group differences over multiple hours, and small sample sizes, which limited statistical power to define a unique peak difference. These groups did not differ from other depressed subjects who had never made a suicide attempt, although if past and future attempters are combined in a single 'any suicide attempt' group, they differ from non-attempters on the AUC measure. The reduced prolactin response in subjects who have made or will make a suicide attempt is consistent with prior research regarding the blunting of response in association with past suicidal behavior (Cleare et al, 1996; Malone et al, 1996; Correa et al, 2000; Duval et al, 2001; Soloff et al, 2003), and also consistent with studies suggesting that prolactin response is a trait measure of serotonergic abnormality not sensitive to changes in clinical state that might indicate an acute risk for suicidal behavior (Flory et al, 1998; Dulchin et al, 2001; Bhagwagar et al, 2002b). As such, it may be a predictor of long-term or lifetime risk for suicidal behavior rather than immediate or short-term risk.

Cortisol responses to FEN were clearly blunted in the future attempters group, and differentiated them from all other groups. This difference was reflected in the peak measure because, unlike prolactin, there was a more defined increase in cortisol response - in all groups except future attempters - that occurred around hour 2 of the procedure, followed by normal morning decline in cortisol levels. Blunting of cortisol response, defined by lower peak change, was related to persistently higher suicidal ideation, even up to a year after the FEN challenge, and greater number of past attempts.

The relationship between cortisol regulation and future suicidal behavior is consistent with a role for HPA dysfunction in suicidal behavior. A recent meta-analysis found that non-suppression of cortisol on the dexamethasone suppression test predicts completed suicide (Mann and Currier, 2007), though a recent follow-up study of the largest such cohort found that this relationship only holds for those with a history of attempt (Jokinen et al, 2007). Higher cortisol levels and dexamethasone resistance characterize melancholia, which carries a heightened risk for suicidal behavior in mood disorders (Grunebaum et al, 2004). On the other hand, lower cortisol responses to administration of combined dexamethasone-corticotropinreleasing factor have been found in suicide attempters 
Table 3 Mood and Behavioral Measures during Challenge Testing

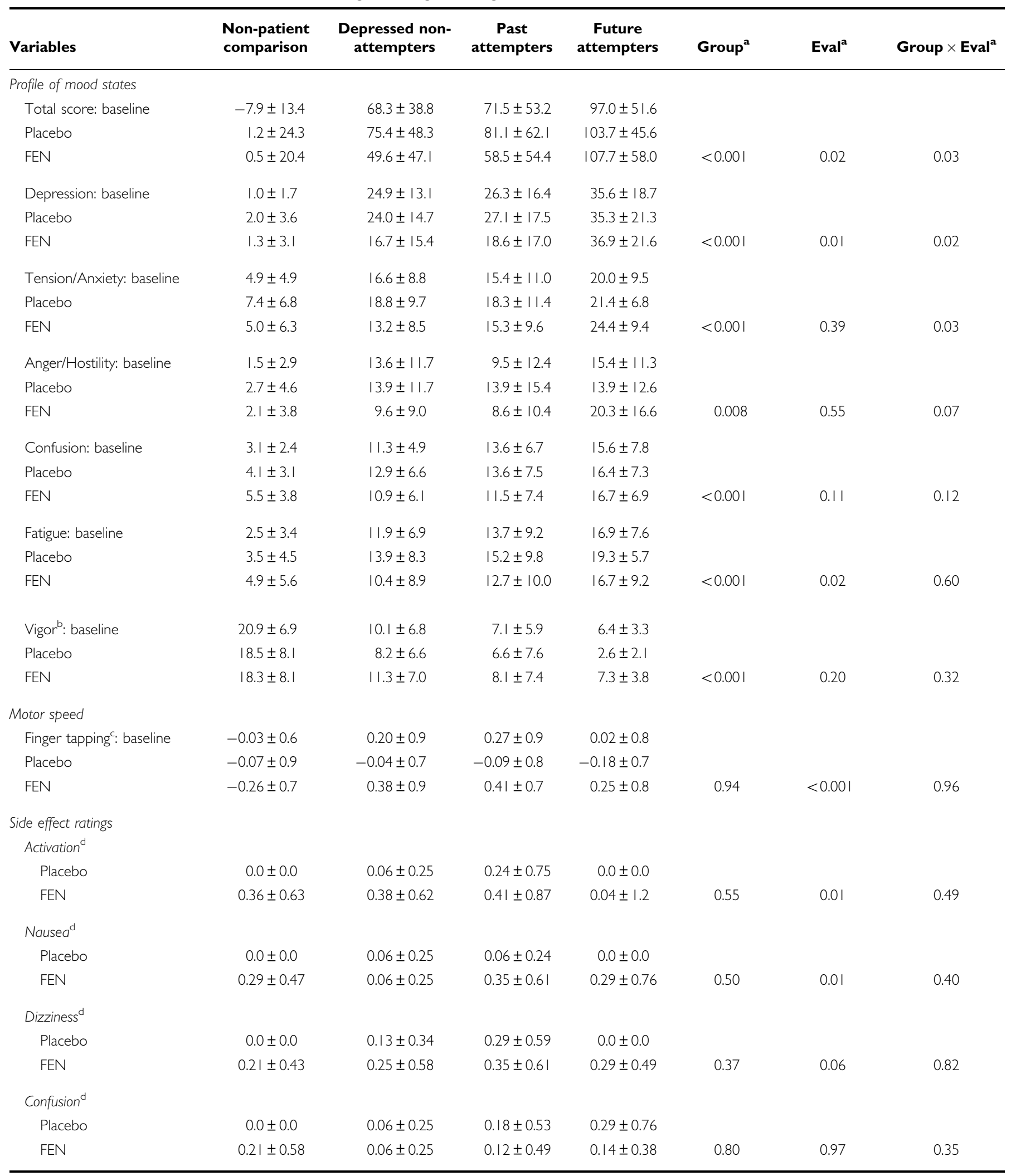

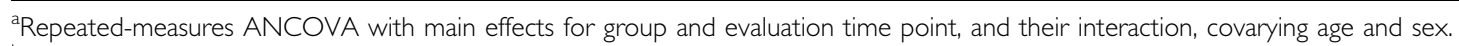

bVigor subscale score positively scaled; all other higher scores correspond to poorer mood.

${ }^{c}$-score relative to population norms, averaged across both hands.

${ }^{\mathrm{d}}$ Not assessed on baseline day since no drug/placebo was administered. 


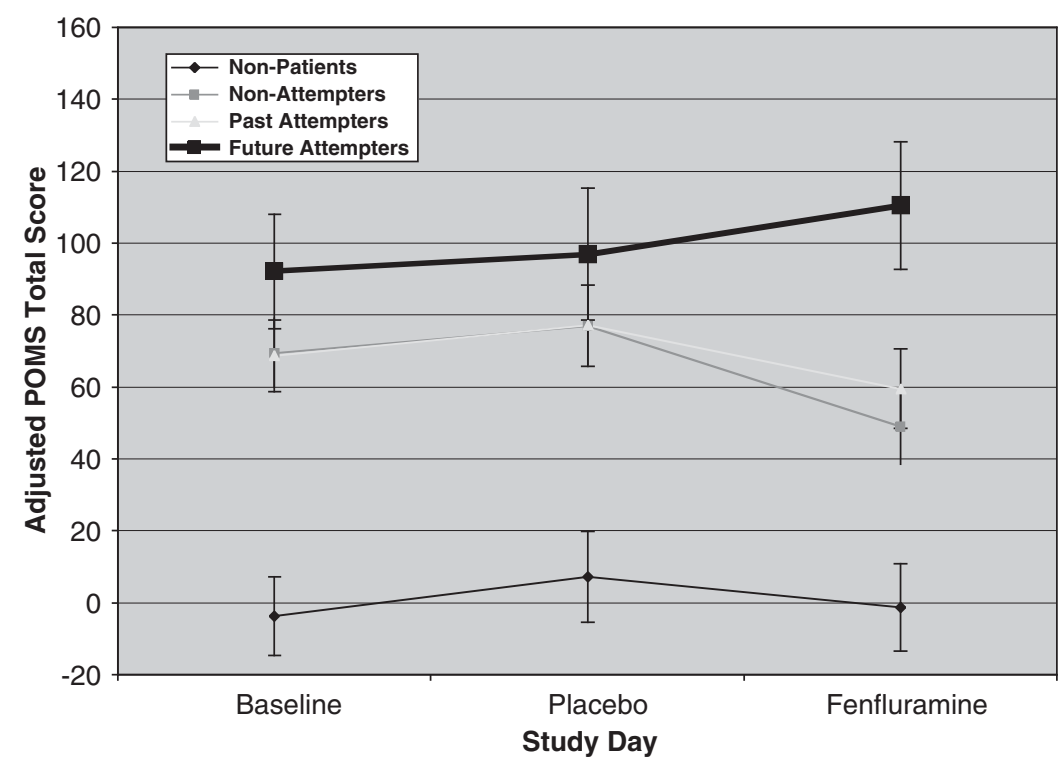

Figure 2 POMS Total score $( \pm$ SEM) on baseline, placebo, and active drug (fenfluramine) days in each subject group, adjusted for age and gender. Decline in Total score (improvement) is significant between placebo and fenfluramine days in non-attempter and past attempter groups, and significantly different than change (worsening) in future attempter group.

(Pfennig et al, 2005), and cortisol responses to pharmacologic challenge tend to be lower in subjects with histories of abuse (Heim and Nemeroff, 2001) or post-traumatic stress disorder (Yehuda, 2001; Oquendo et al, 2003a), both significant risk factors for suicidal behavior (Brodsky et al, 2001; Oquendo et al, 2003b). Cortisol response to pharmacologic challenge appears to be sensitive to clinical state (Bhagwagar et al, 2002b), which may account for some of these discrepancies. Some of these differences may also related to sampling, since the likelihood of future suicide completion even in very high-risk past attempter groups remains small. Alternatively, dysregulation in cortisol does not necessarily imply that levels will always be high or low. One possibility is that ambient levels of cortisol are low and the threshold for response high in subjects at risk for suicidal behavior or with trauma histories, due to hypersensitivity of glucocorticoid receptors and enhanced negative feedback inhibition (Yehuda and LeDoux, 2007). Once that threshold is crossed, however, cortisol responses may be exaggerated.

The post-FEN mood and behavioral response of depressed subjects in this study are consistent with short-term effects of serotonergic medications. Antidepressant effects of serotonergic medications, in terms of improved mood and increased energy, may be evident in the earliest stages of treatment and distinguish those who are likely respond (Taylor et al, 2006). Our data suggest that there may be transient mood effects that are evident within hours after a single dose of a serotonin-enhancing drug. Depressed patients in this study reported a decrease in fatigue and increase in motor speed, and a modest, transient improvement in feelings of depression (30.9\% reduction in score) after receiving FEN. Future attempters, however, also showed evidence of increased energy without an improvement in mood. The combination of being energized without improvement in mood during antidepressant medication treatment may create risk for later suicidality in the context of treatment (Perlis et al, 2007). In our study, logistic regression suggested that absence of mood improvement alone is related to later risk of attempt, but this is at a single, baseline assessment that occurred well before subsequent suicide attempt. Given concerns about the safety of antidepressants, future studies need to evaluate relative effects on mood and activation in the early phases of antidepressant treatment trials and to carefully monitor suicidal thinking to determine if this predicts later risk of suicidal behavior.

The study was limited by its small sample sizes, fixed order of administration of the placebo and FEN to avoid carry-over effects of FEN, and intercurrent PET scanning. There may have been mood and behavioral effects that were more pronounced during the intermediate phases of the study, although analysis of FEN and norfenfluramine levels revealed that levels of both were at or near peak levels even at the final assessment point of the procedure, and prolactin levels remained high at this point as well. Central activity of the drug was likely to have persisted to this point. The PET scans themselves may have had an effect on both prolactin and cortisol response. However, the prolactin and cortisol responses to FEN in all groups on the placebo day showed no increases related to the timing of these scans (prolactin responses were flat and identical in all groups, cortisol responses showed a continuous decline over the $5 \mathrm{~h}$ of the study and were also identical across groups; data available upon request). In addition, prolactin and cortisol responses on the FEN day in non-patients, non-attempters, and past attempters are consistent with prior studies that did not involve scanning. This is the first study to evaluate mood and behavior in the context of a pharmacologic challenge in a prospective sample of suicide attempters, and its results are of interest. However, given the small sample sizes, future studies with larger samples, more frequent mood and behavioral assessments, and counterbalanced order of placebo/drug administration are needed. 
The results of logistic regression, while exploratory, suggest that responses to acute pharmacologic challenge may be useful for generating predictors of later suicidal behavior. Technological and methodological improvements make it feasible to include these measures in larger studies. Cortisol can now be assessed reliably and non-invasively from saliva (Aardal-Eriksson et al, 1998), and alternatives to FEN (ie citalopram) have been used successfully in challenge paradigms (Bhagwagar et al, 2002a; Flory et al, 2004). The ease with which such data can be collected also raise the possibility of developing procedures that might be translated to the clinic, given adequate evaluation of their prognostic value and better understanding of underlying mechanisms relating them to suicidal behavior. Additional research is needed to fully develop these techniques for clinical use.

\section{ACKNOWLEDGEMENTS}

This research was supported by National Institute of Mental Health grants MH-062185 and MH-062155 and the National Association for Research on Schizophrenia and Depression (NARSAD).

\section{DISCLOSURE/CONFLICT OF INTEREST}

Drs Mann and Keilp had received an unrestricted grant from Wyeth-Ayerst pharmaceuticals, the manufacturer of the drug fenfluramine, for a project unrelated to the study described in this paper (cognitive effects of sodium regulation in end-stage hepatic and cardiac failure). All aspects of this study were supported by Public Health Service or foundation grants. All other authors have no conflicts of interest.

\section{REFERENCES}

Aardal-Eriksson E, Karlberg BE, Holm AC (1998). Salivary cortisol-an alternative to serum cortisol determinations in dynamic function tests. Clin Chem Lab Med 36: 215-222.

Barratt ES (1985). Impulsiveness defined within a system model of personality. In: Spielberger CD, Butcher JN (eds). Advances in Personality Assessment, Vol. 5, Earlbaum: New York. pp 113-132.

Barratt ES (1994). Impulsivity and aggression. In: Monahan J, Steadman HJ (eds). Violence and Mental Disorder: Developments in Risk Assessment. University of Chicago Press: Chicago. pp 61-80.

Beck AT, Beck R, Kovacs M (1975). Classification of suicidal behaviors: I. Quantifying intent and medical lethality. $\mathrm{Am}$ J Psychiatry 132: 285-287.

Beck AT, Kovacs M, Weissman A (1979). Assessment of suicidal intention: the Scale for Suicide Ideation. J Consult Clin Psychol 47: 343-352.

Beck AT, Ward CH, Mendelson M, Mock J, Erbaugh J (1961). An inventory for measuring depression. Arch Gen Psychiatry 4: 561-571.

Beck AT, Weissman A, Lester D, Trexler L (1974). The measurement of pessimism: the hopelessness scale. J Consult Clin Psychol 42: 861-865.

Bhagwagar Z, Hafizi S, Cowen PJ (2002a). Acute citalopram administration produces correlated increases in plasma and salivary cortisol. Psychopharmacology (Berlin) 163: 118-120.
Bhagwagar Z, Whale R, Cowen PJ (2002b). State and trait abnormalities in serotonin function in major depression. Br J Psychiatry 180: 24-28.

Brodsky BS, Oquendo M, Ellis SP, Haas GL, Malone KM, Mann JJ (2001). The relationship of childhood abuse to impulsivity and suicidal behavior in adults with major depression. Am J Psychiatry 158: 1871-1877.

Brown GL, Goodwin FK, Ballenger JC, Goyer PF, Major LF (1979). Aggression in humans correlates with cerebrospinal fluid amine metabolites. Psychiatry Res 1: 131-139.

Buss AR, Durkee A (1961). An inventory for assessing different kinds of hostility. J Consult Psychol 21: 343-349.

Cleare AJ, Murray RM, O’Keane V (1996). Reduced prolactin and cortisol responses to D-fenfluramine in depressed compared to healthy matched control subjects. Neuropsychopharmacology 14: 349-354.

Coccaro EF, Siever LJ, Klar HM, Maurer G, Cochrane K, Cooper TB et al (1989). Serotonergic studies in patients with affective and personality disorders. Correlates with suicidal and impulsive aggressive behavior. Arch Gen Psychiatry 46: 587-599.

Correa H, Duval F, Mokrani M, Bailey P, Tremeau F, Staner L et al (2000). Prolactin response to D-fenfluramine and suicidal behavior in depressed patients. Psychiatry Res 93: 189-199.

Dulchin MC, Oquendo MA, Malone KM, Ellis SP, Li S, Mann JJ (2001). Prolactin response to DL-fenfluramine challenge before and after treatment with paroxetine. Neuropsychopharmacology 25: 395-401.

Duval F, Mokrani MC, Correa H, Bailey P, Valdebenito M, Monreal J et al (2001). Lack of effect of HPA axis hyperactivity on hormonal responses to $\mathrm{D}$-fenfluramine in major depressed patients: implications for pathogenesis of suicidal behaviour. Psychoneuroendocrinology 26: 521-537.

First MB, Spitzer RL, Gibbon M, Williams JBW (1997). Structured Clinical Interview for DSM-IV Axis I Disorders, Research Version, Non-patient Edition. New York State Psychiatric Institute: New York, NY.

First MB, Spitzer RL, Gibbon M, Williams JBW, Loma B (1996). Structured Clinical Interview for DSM-IV Axis II Personality Disorders (SCID-II), Version 2.0. Biometric Research Department, New York State Psychiatric Institute: New York.

Flory JD, Mann JJ, Manuck SB, Muldoon MF (1998). Recovery from major depression is not associated with normalization of serotonergic function. Biol Psychiatry 43: 320-326.

Flory JD, Manuck SB, Perel JM, Muldoon MF (2004). A comparison of D, L-fenfluramine and citalopram challenges in healthy adults. Psychopharmacology 174: 376-380.

Grunebaum MF, Galfalvy HC, Oquendo MA, Burke AK, Mann JJ (2004). Melancholia and the probability and lethality of suicide attempts. Br J Psychiatry 184: 534-535.

Grunebaum MF, Keilp J, Li S, Ellis SP, Burke AK, Oquendo MA et al (2005). Symptom components of standard depression scales and past suicidal behavior. J Affect Disord 87: 73-82.

Hamilton M (1960). A rating scale for depression. J Neurol Neurosurg Psychiatry 23: 56-62.

Heim C, Nemeroff CB (2001). The role of childhood trauma in the neurobiology of mood and anxiety disorders: preclinical and clinical studies. Biol Psychiatry 49: 1023-1039.

Jokinen J, Carlborg A, Martensson B, Forslund K, Nordstrom AL, Nordstrom P (2007). DST non-suppression predicts suicide after attempted suicide. Psychiatry Res 150: 297-303.

Kegeles LS, Malone KM, Slifstein M, Ellis SP, Xanthopoulos E, Keilp JG et al (2003). Response of cortical metabolic deficits to serotonergic challenge in familial mood disorders. $\mathrm{Am}$ J Psychiatry 160: 76-82.

Malone KM, Corbitt EM, Li S, Mann JJ (1996). Prolactin response to fenfluramine and suicide attempt lethality in major depression. Br J Psychiatry 168: 324-329. 
Mann JJ (2003). Neurobiology of suicidal behaviour. Nat Rev Neurosci 4: 819-828.

Mann JJ, Currier D (2007). A review of prospective studies of biologic predictors of suicidal behavior in mood disorders. Arch Suicide Res 11: 3-16.

Mann JJ, Waternaux C, Haas GL, Malone KM (1999). Toward a clinical model of suicidal behavior in psychiatric patients. Am J Psychiatry 156: 181-189.

Manuck SB, Flory JD, McCaffery JM, Matthews KA, Mann JJ, Muldoon MF (1998). Aggression, impulsivity, and central nervous system serotonergic responsivity in a nonpatient sample. Neuropsychopharmacology 19: 287-299.

Manuck SB, Flory JD, Muldoon MF, Ferrell RE (2002). Central nervous system serotonergic responsivity and aggressive disposition in men. Physiol Behav 77: 705-709.

McNair DM, Lorr M, Droppleman LF (1981). Manual for the Profile of Mood States. Educational and Industrial Testing Service: San Diego.

New AS, Trestman RF, Mitropoulou V, Goodman M, Koenigsberg $\mathrm{HH}$, Silverman J et al (2004). Low prolactin response to fenfluramine in impulsive aggression. J Psychiatr Res 38: 223-230.

Newman ME, Shapira B, Lerer B (1998). Evaluation of central serotonergic function in affective and related disorders by the fenfluramine challenge test: a critical review. Int J Neuropsychopharmcol 1: 49-69.

Oquendo MA, Echavarria G, Galfalvy HC, Grunebaum MF, Burke A, Barrera A et al (2003a). Lower cortisol levels in depressed patients with comorbid post-traumatic stress disorder. Neuropsychopharmacology 28: 591-598.

Oquendo MA, Friend JM, Halberstam B, Brodsky BS, Burke AK, Gruenbaum MF et al (2003b). Association of comorbid posttraumatic stress disorder and major depression with greater risk for suicidal behavior. Am J Psychiatry 160: 580-582.

Oquendo MA, Halberstam B, Mann JJ (2003c). Risk factors for suicidal behavior: the utility and limitations of research instruments. In: First M (ed). Standardized Evaluation in
Clinical Practice, Review of Psychiatry, Vol. 22, American Psychiatric Publishing: Washington, DC. pp 103-130.

Oquendo MA, Placidi GP, Malone KM, Campbell C, Keilp J, Brodsky B et al (2003d). Positron emission tomography of regional brain metabolic responses to a serotonergic challenge and lethality of suicide attempts in major depression. Arch Gen Psychiatry 60: 14-22.

Perlis RH, Beasley Jr CM, Wines Jr JD, Tamura RN, Cusin C, Shear D et al (2007). Treatment-associated suicidal ideation and adverse effects in an open, multicenter trial of fluoxetine for major depressive episodes. Psychother Psychosom 76: 40-46.

Pfennig A, Kunzel HE, Kern N, Ising M, Majer M, Fuchs B et al (2005). Hypothalamus-pituitary-adrenal system regulation and suicidal behavior in depression. Biol Psychiatry 57: 336-342.

Pruessner JC, Kirschbaum C, Meinlschmid G, Hellhammer DH (2003). Two formulas for computation of the area under the curve represent measures of total hormone concentration $v \boldsymbol{s}$ time-dependent change. Neuropsychoendocrinology 28: 916-931.

Reitan RM, Wolfson D (1985). The Halstead-Reitan Neuropsychological Test Battery. Neuropsychology Press: Tucson, Arizona.

Soloff PH, Kelly TM, Strotmeyer SJ, Malone KM, Mann JJ (2003). Impulsivity, gender, and response to fenfluramine challenge in borderline personality disorder. Psychiatry Res 119: 11-24.

Spitzer RL, Williams JBW, Gibbon M, First MB (1990). Instruction Manual for the Structured Clinical Interview for the DSM-IV (SCID-P). American Psychiatric Press: Washington, DC.

Spreen O, Strauss E (1998). A Compendium of Neuropsychological Tests. Oxford University Press: New York.

Taylor MJ, Freemantle N, Geddes JR, Bhagwagar Z (2006). Early onset of selective serotonin reuptake inhibitor antidepressant action: systematic review and meta-analysis. Arch Gen Psychiatry 63: $1217-1223$.

Yehuda R (2001). Biology of posttraumatic stress disorder. J Clin Psychiatry 62: 41-46.

Yehuda R, LeDoux J (2007). Response variation following trauma: a translational neuroscience approach to understanding PTSD. Neuron 56: 19-32. 